\title{
HETEROZYGOUS HEMOCHROMATOSIS: A RARE CASE REPORT
}

\author{
Nirmit Patel ${ }^{1}$, Aneri Patel ${ }^{1}$, Viral Patel ${ }^{2}$, Sakshar Patel $^{3}$, Dhrushi Patel $^{4}$, Parth Patel ${ }^{4}$, \\ Sarth Patel ${ }^{4}$, and Manan Patel ${ }^{5}$ \\ ${ }^{1}$ Gujarat Cancer Society Medical College \\ ${ }^{2}$ Medical College Baroda \\ ${ }^{3}$ Smt NHL Municipal Medical College \\ ${ }^{4}$ BJ Medical College \\ ${ }^{5}$ Pacific Medical College \& Hospital
}

July 26, 2021

\begin{abstract}
To our knowledge,this is the first report of $31 \mathrm{Y} \mathrm{F}$ has no family history and usually,patients do not manifest symptoms if they are heterozygous.Individuals heterozygous for $\mathrm{C} 282 \mathrm{Y}$ who have a coexisting insult to the liver,associated with the use of medications,may present with overt manifestations of iron overload.
\end{abstract}

\section{HETEROZYGOUS HEMOCHROMATOSIS: A RARE CASE REPORT}

Dr.Nirmit Patel 1,MBBS;Dr Aneri Patel 1,MBBS:,Dr Viral Patel 2,MBBS;Dr Sakshar Patel 3,MBBS; Dr Dhrushi Patel 4,MBBS;Dr Parth Patel 4,MBBS;Dr Sarth Patel 4,MBBS;

Dr.Manan Patel 5,MBBS.

1.GCS Medical College,Hospital \& Research Center,Ahmedabad 2.Medical College,Baroda 3.NHL Medical college,Ahmedabad 4.B.J Medical college,Ahmedabad 5. Pacific Medical College and Hospital,Udaipur

\section{ABSTRACT:}

Hereditary hemochromatosis $(\mathrm{HH})$ is a common autosomal recessive iron storage disease. The classic clinical triad of liver cirrhosis,hyperpigmentation, and diabetes is nowadays rare, most likely due to early recognition1. Usually, the homozygous C282Y mutation in the HFE gene is responsible for most cases of hereditary hemochromatosis2. Here, we are presenting a case of a 31 Year Old female patient, who presented with yellow discoloration of her urine followed by yellow discoloration of her eyes associated with headache, nausea, and vomiting and later on, it was diagnosed heterozygous hemochromatosis.

\section{BACKGROUND:}

Hereditary hemochromatosis (HH), is an autosomal recessive disorder with iron overload in several organs, especially within the liver. The monoallelic genetic disorder-hereditary hemochromatosis, was first described by Trousseau in 1889 as a triad of glycosuria, cirrhosis, and hyperpigmentation of the skin. The term hemochromatosis was first employed by Von Recklinghausen in 18893. HH is usually because of two histone family $\mathrm{E} 1$ (HFE1), gene mutations-C282Y, and H63D4. The HFE gene is present within the human leukocyte antigen (HLA) class 1 region on chromosome 6 between the genes coding for HLA-A and HLA-B. Two mutations within the HFE gene are described. The first mutation is at C282Y,consisting of substitution 
of tyrosine for cysteine at amino acid position 282. The second mutation is at H63D in which amino acid is substituted for histidine in position 63. Either C282Y or H63D is found in most patients with HH5. Secondary hemochromatosis is caused by disorders of erythropoiesis and treatment of the diseases with blood transfusions6. Hereditary hemochromatosis is characterized by abnormal iron absorption from the diet leading to progressive iron overload, causing tissue damage in several organs, particularly the liver7.HFE gene mutations are strongly associated with predisposition to $\mathrm{HH}$ and are also implicated in other disorders such as rheumatoid arthritis, type $2 \mathrm{DM}$, porphyria cutanea tarda, and coronary heart condition. Considerable ethnic variation is observed within the distribution of HFE mutations. We hereby report a case of a heterozygous hemochromatosis who presented with a yellow discoloration of her urine followed by yellow discoloration of her eyes associated with headache, nausea, and vomiting8.

\section{CASE DESCRIPTION:}

31 Year Old, otherwise healthy female with no significant past medical history presented to ED with complaints of yellow discoloration of her urine followed by yellow discoloration of her eyes associated with headache, nausea, and vomiting.She also had abnormally high liver enzymes with AST and ALT as high as the 2000's with an associated conjugated hyperbilirubinemia. Before that, she had decreased appetite and some vague abdominal pain for the past 2 days along with the sensation of constipation. She took a probiotic and some cranberry pills which didn't seem to help improve her urine color and abdominal discomfort. Family history was negative for liver disease and autoimmune disorders. She denies a history of IV drug use, recent NSAIDs use, and recreational medications such as ecstasy or amphetamines or cocaine. All the laboratory workup for infections as well as autoimmune etiology were negative. She had an Ophthalmology/optometry examination in August 2019 and no Kayser-Fleischer rings were seen. USG abdomen and MRCP shows gallbladder wall thickening with gallbladder wall measuring up to $4.5 \mathrm{~mm}$ and no cholelithiasis/choledocholithiasis. Her gamma globulin level was normal, Monospot negative, negative for hepatitis A, B, and C and HIV screen. Liver enzymes remain elevated $(>1000)$ in a predominantly hepatocellular pattern with cholestasis and decided to do the outpatient liver biopsy next week and discharged, but the Patient began to develop RUQ pain, nausea, vomiting, and worsening jaundice yesterday which prompted her to return to ED. To differentiate the suspicious cause of conjugated hyperbilirubinemia with transaminitis in 1000s we decided to do a liver biopsy.A liver Biopsy was performed and it demonstrated subtotal hepatic necrosis with severe inflammation and a very small amount of iron and fibrosis in the liver (Table 2). PCR of blood identifies a single mutation $\mathrm{C} 282 \mathrm{Y}$ identified. She is being treated with phlebotomy and her liver enzymes improve significantly.

Table 2: 


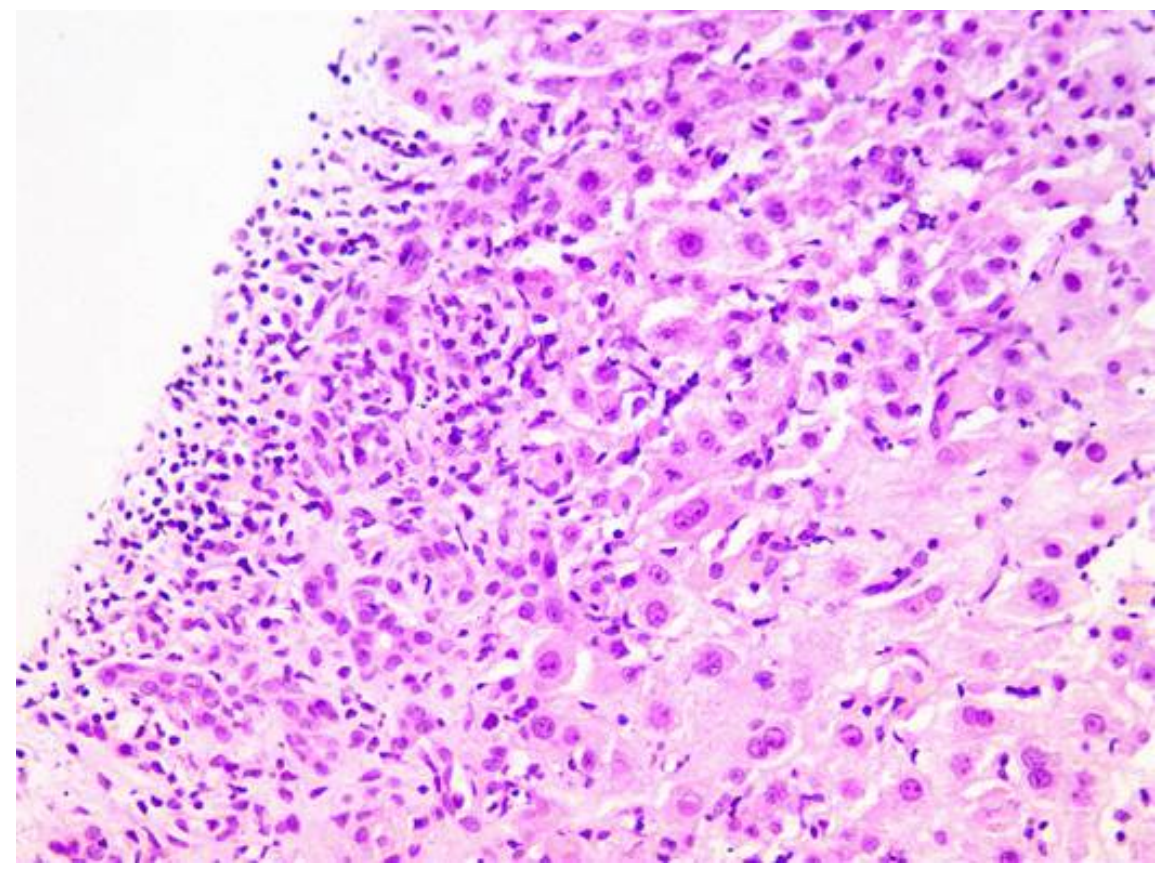

Figure 1: Individual cell apoptosis appreciated in Lobules. No Granulomas or fibrosis appreciated

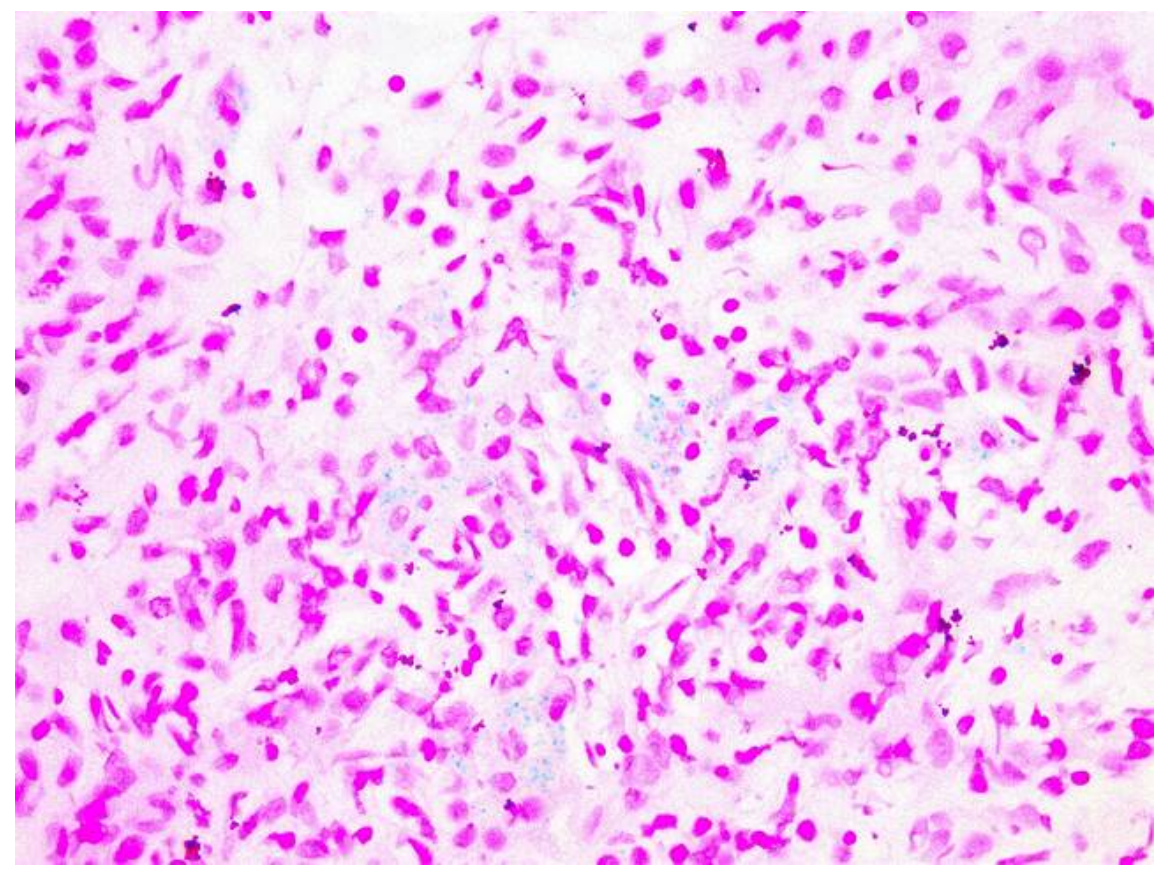

Figure 2: Positive Iron stain 


\section{CASE DISCUSSION:}

$\mathrm{HH}$ are often either asymptomatic or symptomatic. In some cases who test positive for HH remain asymptomatic throughout their life. In the present case, the patient presented with icterus, yellowish discoloration of urine, nausea, vomiting, and negative family history. The diagnosis of $\mathrm{HH}$ is predicated on the measurement of transferrin saturation, serum ferritin levels, and mutation analysis of HFE. In the present case, total iron $(326 \mathrm{mg} / \mathrm{dL})$ and serum ferritin $(2506 \mathrm{mg} / \mathrm{dL})$ were increased with a marked increase in transferrin saturation- $(88 \%),>1.9$ iron-age-index which is diagnostic of hemochromatosis. The patient had conjugated hyperbilirubinemia with transaminitis, a standard clinical finding in hemochromatosis. Liver biopsy confirmed the deposition of iron. Liver biopsy is helpful to evaluate the underlying disease which determines the fibrosis and degree of iron load9. The importance of liver biopsy also lies in the proven fact that documentation of extensive bridging fibrosis or cirrhosis includes a profound impact on the prognosis in $\mathrm{HH}$ patients 10. The absence of symptoms of hemochromatosis is common, particularly in young individuals, just because of the variable phenotypic expression of the disease and variations of lifetime accumulation of iron stores in tissues. Early detection, in conjunction with various routine screening procedures, is helpful and also important because effective therapy is accessible through phlebotomy 10-11-12. In patients with hidden liver dysfunction, search for underlying causes, like hemochromatosis, especially within the presence of skin pigment changes, recent-onset diabetes, or cardiomyopathy. Individuals who are heterozygous for $\mathrm{C} 282 \mathrm{Y}$ and who have a coexisting insult to the liver, including medicine abuse, may present with overt manifestations of hemochromatosis. In hemochromatosis, a traditional anticipation are often achieved if early diagnosis and treatment are given before irreversible damage can occur.

\section{CONCLUSION:}

Hemochromatosis can be detected incidentally by routine examination and blood analysis, and its diagnosis can be made easily by mutation analysis. Today, C282Y and H63D mutations are detected in whole blood by polymerase chain reaction (PCR). Accumulation of iron in the body and detection of homozygous $\mathrm{C} 282 \mathrm{Y}$ mutation is sufficient for the diagnosis of the disease.C282Y homozygosity is the most common mutation for hemochromatosis and proceeds with maximum iron accumulation. While the patients with both $\mathrm{C} 282 \mathrm{Y} / \mathrm{H} 63 \mathrm{D}$ heterozygosity show moderate iron accumulation, H63D homozygous, and C282Y heterozygous are usually normal. To prevent the complication and for early detection, some specialists suggest screening to detect HH before it causes problems 10-11-12. To our best knowledge, this is the first case of its kind of hemochromatosis in which the patient presented with symptomatic hepatic failure without any positive family history in the setting of a single $\mathrm{C} 282 \mathrm{Y}$ gene mutation 13 . Obstructive cause were the initial suspected etiology and the most common etiology being ruled out via imaging and laboratory test.The team of Gastroenterology and Oncomedicine were refused that this level of iron overload as secondary to hemochromatosis being caused by just the single gene involvement 14. We conclude that individuals who may be heterozygous forC282Y gene who have coexisting insult to the liver, associated with the use of medications may still present with overt manifestations of iron overload 14.

\section{REFERENCE:}

1. Milman, N., Clausen, J. O., \& Jordal, R. (1995). Iron status in young Danish men and women: a population survey comprising 548 individuals. Annals of Hematology, 70(4), 215-221. https: //doi.org/10.1007/bf01700378

2. Aguilar Martinez, P., Jeanjean, P., Masmejean, C., Guillard, A., Biron, C., Rabesandratana, H., \& Schved, J. (1997). Simple and Rapid Detection of the Newly Described Mutations in the HLA-H Gene. Blood, 89(5), 1835-1836. https://doi.org/10.1182/blood.v89.5.1835.1835_1835_1836

3. Poddar, S. (2006). Hereditary Hemochromatosis-Special Reference to Indian Scenario. International Journal of Human Genetics, 6(1), 73-79. https://doi.org/10.1080/09723757.2006.11885947

4. Panigrahi, I., Ahmad, F., Kapoor, R., Sharma, P., Makharia, G., \& Saxena, R. (2006). Evidence for non-HFE linked hemochromatosis in Asian Indians. Indian Journal of Medical Sciences, 60(12), 491. https://doi.org/10.4103/0019-5359.28978 
5. Capell, P. (2004). Case Study: Hemachromatosis in Type 2 Diabetes. Clinical Diabetes, 22(2), 101102. https://doi.org/10.2337/diaclin.22.2.101

6. Gattermann, N. (2009). The Treatment of Secondary Hemochromatosis. Deutsches Aerzteblatt Online. Published. https://doi.org/10.3238/arztebl.2009.0499

7. Baynes, R. D., Cook, J. D., Bothwell, T. H., Friedman, B. M., \& Meyer, T. E. (1994). Serum transferrin receptor in hereditary hemochromatosis and African siderosis. American Journal of Hematology, 45(4), 288-292. https://doi.org/10.1002/ajh.2830450404

8. Dhillon, B. K. (2007). Frequency of primary iron overload and HFE gene mutations (C282Y, H63D and $\mathrm{S} 65 \mathrm{C}$ ) in chronic liver disease patients in north India. World Journal of Gastroenterology, 13(21), 2956. https://doi.org/10.3748/wjg.v13.i21.2956

9. Bacon, B. R., Adams, P. C., Kowdley, K. V., Powell, L. W., \& Tavill, A. S. (2011). Diagnosis and management of hemochromatosis: 2011 Practice Guideline by the American Association for the Study of Liver Diseases. Hepatology, 54(1), 328-343. https://doi.org/10.1002/hep. 24330

10. Whitlock, E. P., Garlitz, B. A., Harris, E. L., Beil, T. L., \& Smith, P. R. (2006). Screening for Hereditary Hemochromatosis: A Systematic Review for the U.S. Preventive Services Task Force. Annals of Internal Medicine, 145(3), 209. https://doi.org/10.7326/0003-4819-145-3-200608010-00009

11. Tavill, A. (2001). Diagnosis and management of hemochromatosis. Hepatology, 33(5), 1321-1328. https://doi.org/10.1053/jhep.2001.24783

12. Kj, A., Lc, G., Cc, C., Nj, O., Mb, D., Aj, N., Ce, M., M, B., Ae, N., Cd, V., Gj, A., Mc, S., Gg, G., Dr, E., Jl, H., Jk, O., Lw, P., \& Dm, G. (2008, January 1). Iron-overload-related disease in HFE hereditary hemochromatosis. Abstract - Europe PMC. https://europepmc.org/article/med/18199861

13. Doran, T., Bashir, H., Trejaut, J., Bassett, M., Halliday, J., \& Powell, L. (1981). Idiopathic haemochromatosis in the Australian population: HLA linkage and recessivity. Human Immunology, 2(3), 191-200. https://doi.org/10.1016/0198-8859(81)90011-2

14. Marx, J. (1997, August 1). Iron deficiency in. . . European Journal of Clinical Nutrition. https://www.nature.com/articles/1600440?error=cookies_not_supported\&code= 6a1085d7-63a2-4776-bae8-b90a2bd41fce 